\title{
Peak Width of Skeletonized Mean Diffusivity as Neuroimaging Biomarker in Cerebral Amyloid Angiopathy
}

\author{
(D) N. Raposo, (D) M.C. Zanon Zotin, (D). Schoemaker, (D). Xiong, (DP. Fotiadis, (D)A. Charidimou, (D). Pasi, (D). Boulouis,
}

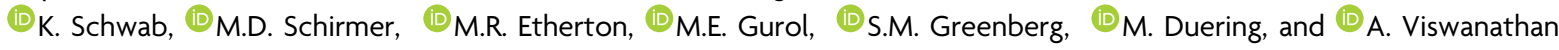

\section{ABSTRACT}

BACKGROUND AND PURPOSE: Whole-brain network connectivity has been shown to be a useful biomarker of cerebral amyloid angiopathy and related cognitive impairment. We evaluated an automated DTI-based method, peak width of skeletonized mean diffusivity, in cerebral amyloid angiopathy, together with its association with conventional MRI markers and cognitive functions.

MATERIALS AND METHODS: We included 24 subjects (mean age, 74.7 [SD, 6.0] years) with probable cerebral amyloid angiopathy and mild cognitive impairment and 62 patients with $\mathrm{MCl}$ not attributable to cerebral amyloid angiopathy (non-cerebral amyloid angiopathy-mild cognitive impairment). We compared peak width of skeletonized mean diffusivity between subjects with cerebral amyloid angiopathy-mild cognitive impairment and non-cerebral amyloid angiopathy-mild cognitive impairment and explored its associations with cognitive functions and conventional markers of cerebral small-vessel disease, using linear regression models.

RESULTS: Subjects with Cerebral amyloid angiopathy-mild cognitive impairment showed increased peak width of skeletonized mean diffusivity in comparison to those with non-cerebral amyloid angiopathy-mild cognitive impairment $(P<.001)$. Peak width of skeletonized mean diffusivity values were correlated with the volume of white matter hyperintensities in both groups. Higher peak width of skeletonized mean diffusivity was associated with worse performance in processing speed among patients with cerebral amyloid angiopathy, after adjusting for other MRI markers of cerebral small vessel disease. The peak width of skeletonized mean diffusivity did not correlate with cognitive functions among those with non-cerebral amyloid angiopathy-mild cognitive impairment.
\end{abstract}

CONCLUSIONS: Peak width of skeletonized mean diffusivity is altered in cerebral amyloid angiopathy and is associated with performance in processing speed. This DTI-based method may reflect the degree of white matter structural disruption in cerebral amyloid angiopathy and could be a useful biomarker for cognition in this population.

ABBREVIATIONS: $\mathrm{CAA}=$ cerebral amyloid angiopathy; $\mathrm{CMB}=$ cerebral microbleeds; $\mathrm{CSO}-\mathrm{PVS}=$ perivascular spaces in the centrum semiovale; $\mathrm{CSS}=$ cortical superficial siderosis; $C S V D=$ cerebral small vessel disease; $I C H=$ intracerebral hemorrhage; $I Q R=$ interquartile range; $\mathrm{MCl}=$ mild cognitive impairment; $\mathrm{MD}=$ mean diffusivity; MMSE = Mini-Mental State Examination; PSMD = peak width of skeletonized mean diffusivity; nTBV = normalized total brain volume; $\mathrm{nWMHV}=$ normalized white matter hyperintensity volume; $\mathrm{WMH}=$ white matter hyperintensities

S

poradic cerebral amyloid angiopathy (CAA) is a highly prevalent cerebral small-vessel disease (cSVD) in the elderly. ${ }^{1}$ CAA is a well-known cause of lobar intracerebral hemorrhage (ICH) and is also increasingly recognized as a major contributor to vascular cognitive impairment and dementia. ${ }^{2,3}$ Although underlying
Received July 17, 2020; accepted after revision November 20.

From the Stroke Research Center (N.R., M.C.Z.Z., D.S., L.X., P.F., A.C., K.S., M.D.S., M.R.E., M.E.G., S.M.G., A.V.), Massachusetts General Hospital, Harvard Medical School, Boston, Massachusetts; Department of Neurology (N.R.), Centre Hospitalier Universitaire de Toulouse, Toulouse, France; Toulouse Neurolmaging Center (N.R.), Université de Toulouse, Institut National de la Santé et de la Recherche Médicale, Toulouse, Université Paul Sabatier, France; Center for Imaging Sciences and Medical Physics (M.C.Z.Z.). Department of Medical Imaging, Hematology and Clinical Oncology, Ribeirão Preto Medical School, University of São Paulo, Ribeirão Preto, São Paulo, Brazil; Department of Neurology (M.P.), Centre Hospitalier Universitaire de Lille, Lille, France; Department of Neuroradiology (G.B.), Centre Hospitalier Sainte-Anne, Université Paris-Descartes, Paris, France; Computer Science and Artificial Intelligence Lab (M.D.S.), Massachusetts Institute of Technology, Boston, Massachusetts; Department of Population Health Sciences (M.D.S.), German Center for Neurodegenerative Diseases, Bonn, Germany; and Medical Image Analysis Center (MIAC) and Quantitative Biomedical Imaging Group (M.D.), Department of Biomedical Engineering, University of Basel, Basel, Switzerland.
N. Raposo and M.C. Zanon Zotin contributed equally to this work.

This study was supported by the following National Institutes of Health grants: R01AG047975, R01AG026484, P50AG005134, K23AG02872605. Nicolas Raposo was supported by a Fulbright Scholarship and received an Arthur Sachs Scholarship from the Harvard University Committee on General Scholarship and a Philippe Foundation research grant. Markus D. Schirmer was supported by the European Union's Horizon 2020 Research and Innovation Program under the Marie Sklodowska-Curie grant agreement No 753896.

Please address correspondence to Nicolas Raposo, MD, PhD, Service de Neurologie, Hôpital Pierre-Paul Riquet, Centre Hospitalier Universitaire de Toulouse, Place Baylac 31059 Toulouse Cedex 9, France; e-mail: raposo.n@chu-toulouse.fr

- Indicates open access to non-subscribers at www.ajnr.org

Indicates article with online supplemental data.

http://dx.doi.org/10.3174/ajnr.A7042 
mechanisms leading to cognitive impairment in CAA remain uncertain, it has been hypothesized that recurrent vascular lesions cause progressive disruption of the brain's structural connectivity, compromising network efficiency. ${ }^{4,5}$ Conventional MR imaging markers of CAA, including lobar cerebral microbleeds (CMB), ${ }^{6}$ cortical superficial siderosis (cSS), ${ }^{7}$ white matter hyperintensities (WMH $),{ }^{8}$ and cortical microinfarcts ${ }^{9}$ have been linked to cognitive functions. However, these associations are mostly weak and inconsistent across studies, suggesting that these markers may reflect only the tip of the iceberg in the whole spectrum of vascular pathology. ${ }^{10}$

Accumulating evidence suggests that DTI methods detect loss of microstructural integrity and other abnormalities not captured by structural MRI and tend to show stronger associations with cognition in subjects with cSVD. ${ }^{11,12}$ Yet, the direct application of DTI in routine clinical practice is hampered by highly variable, complex, and time-consuming processing techniques.

Peak width of skeletonized mean diffusivity (PSMD) is a recently developed, fully automated DTI marker based on the skeletonization of white matter tracts and histogram analysis of mean diffusivity (MD). ${ }^{13}$ PSMD has been shown to be particularly sensitive to vascular-related white matter abnormalities, demonstrating consistent associations with processing speed in subjects with cSVD. ${ }^{13}$ However, despite the common nature and high prevalence of CAA in aging populations, potential applications of PSMD in CAA have been scarcely investigated.

In the current study, we tested whether PSMD reflects the burden of underlying cSVD and cognitive dysfunctions in subjects with CAA. Among subjects with mild cognitive impairment (MCI) recruited specifically from a memory clinic setting, we explored the following: 1) whether PSMD is increased in subjects with CAA compared with those with non-CAA, 2) whether it is associated with structural MRI markers of CAA, and 3) whether it is correlated with cognitive functions.

\section{MATERIALS AND METHODS}

The data supporting findings of this study are available from the corresponding author on reasonable request.

\section{Participants}

We analyzed data from a memory clinic research cohort from the Massachusetts General Hospital between March 2010 and October 2016 and designed a case-control study. Patients underwent clinical examination, neuropsychological evaluation, and research MRI. The Institutional Review Board of Massachusetts General Hospital approved this study, and written informed consent was obtained from all participants or their surrogates.

We included subjects 55 years of age or older meeting Petersen criteria (2004) ${ }^{14}$ for MCI based on clinical assessment of functional status, neurologic evaluation, and extensive neuropsychological assessment. On visual examination of research MRIs, patients with MCI were categorized as the following: 1) CAA-MCI if they fulfilled the Modified Boston criteria ${ }^{15}$ for probable CAA (55 years of age or older; multiple lobar CMB with or without cSS or a single lobar cerebral microbleed and the presence of cSS), or 2) non-CAA-MCI. In both groups, exclusion criteria were dementia, history of symptomatic or asymptomatic ICH (defined as hemorrhagic focus of $>5$ $\mathrm{mm}$ in diameter), the presence of deep CMB (suggesting arteriolosclerosis as underlying cSVD), contraindications for MRI, and the presence of excessive motion artifacts on DTI on careful qualitative visual inspection.

\section{Data Collection}

We systematically collected demographic information and medical history for each participant. All subjects underwent a standardized neuropsychological test battery, as previously described. ${ }^{9}$ Global cognitive status was assessed with the Mini-Mental State Examination (MMSE). ${ }^{16}$ Performance on neuropsychological tests was clustered to create composite scores exploring specific cognitive domains: ${ }^{17}$ executive function (Trail-Making Test $\mathrm{B}^{18}$ and Digit Span Backward ${ }^{19}$ ), processing speed/attention (Trail-Making Test $A,{ }^{20}$ Digit Span Forward, and the Wechsler Adult Intelligence Scale, Third Edition Digit Symbol Coding ${ }^{19}$ ), memory (Hopkins Verbal Learning Test-Revised ${ }^{21}$ and Wechsler Memory Scale for logical memory, ${ }^{22}$ immediate recall, and delayed recall), and language function (Boston Naming Test $^{23}$ and Animal Naming $\left.\mathrm{Test}^{24}\right)$. Performance on each test was first transformed into sex-, age-, and education-adjusted $z$ scores using published normative data. ${ }^{16,24-26}$ Then, the $z$ scores were averaged within each composite domain to obtain domain-specific scores for each subject.

\section{MRI Acquisition}

Neuroimaging was acquired on a 3T MRI scanner (TIM Trio; Siemens), using a 32-channel head coil. MRI sequences included high-resolution diffusion-weighted imaging (60 directions; TR = $8040 \mathrm{~ms}$; TE $=84 \mathrm{~ms}$; slice thickness $=2 \mathrm{~mm}$; in-plane resolution $=2 \times 2 \mathrm{~mm}$; b-value $\left.=700 \mathrm{~s} / \mathrm{mm}^{2}\right)$, 3D T1-weighted multiecho $(\mathrm{TR}=2300 \mathrm{~ms}$; $\mathrm{TE}=2.98 \mathrm{~ms}$; slice thickness $=1 \mathrm{~mm}$; inplane resolution $=1 \times 1 \mathrm{~mm})$, 3D FLAIR $(\mathrm{TR}=6000 \mathrm{~ms}$; TE $=$ $455 \mathrm{~ms}$; slice thickness $=1 \mathrm{~mm}$; in-plane resolution $=1 \times 1 \mathrm{~mm}$ ), and SWI $(\mathrm{TR}=27 \mathrm{~ms}$; TE $=20 \mathrm{~ms}$; slice thickness $=1.5 \mathrm{~mm}$; inplane resolution $=0.86 \times 0.86 \mathrm{~mm}$ ).

The median delay between neuropsychological evaluation and MRI was 1.85 months (interquartile range $[\mathrm{IQR}]=0.00-3.06$ months) and was shorter in subjects with CAA-MCI compared with those with non-CAA-MCI (median, 0 [IQR $=0.0-0.24$ ] months versus 2.1 [1.17-3.30] months; $P<.001$ ).

\section{DTI and PSMD Processing}

PSMD was calculated from unprocessed DTI data using a publicly available script (PSMD Marker, Version1.0; http://www.psmdmarker.com). ${ }^{13}$ This fully automated pipeline relies on the FMRIB Software Library (FSL, Version 6.0.1; http://www.fmrib.ox.ac.uk/ fsl) for the preprocessing of DTI data (eddy current and motion correction, [eddy_correct https://fsl.fmrib.ox.ac.uk/fsl/fslwiki/ eddy]), brain extraction (FSL Brain Extraction tool; http://fsl.fmrib. ox.ac.uk/fsl/fslwiki/BET), and tensor fitting (dtifit; http://fsl.fmrib. ox.ac.uk/fsl/fsl-4.1.9/fdt/fdt_dtifit.html), followed by skeletonization of preprocessed DTI data, application of a custom mask, and histogram analysis (Fig 1). Precisely, DTI data were skeletonized using the Tract-Based Spatial Statistics procedure (TBSS; http://fsl. fmrib.ox.ac.uk/fsl/fslwiki/TBSS), part of the FSL, and the FMRIB 1-mm fractional anisotropy template (FA template; https://fsl. fmrib.ox.ac.uk/fsl/fslwiki/Atlases, thresholded at a fractional 

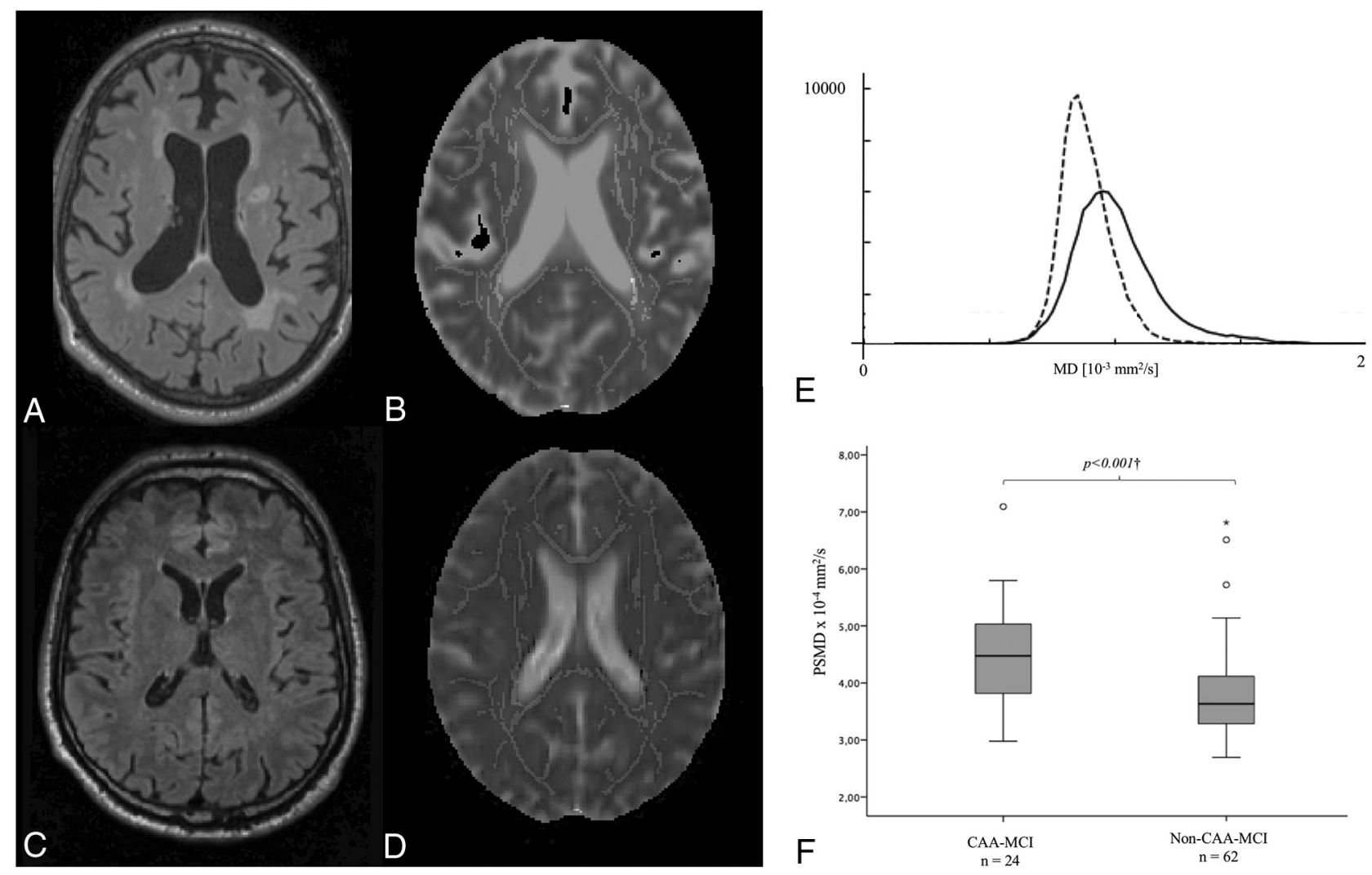

$E$

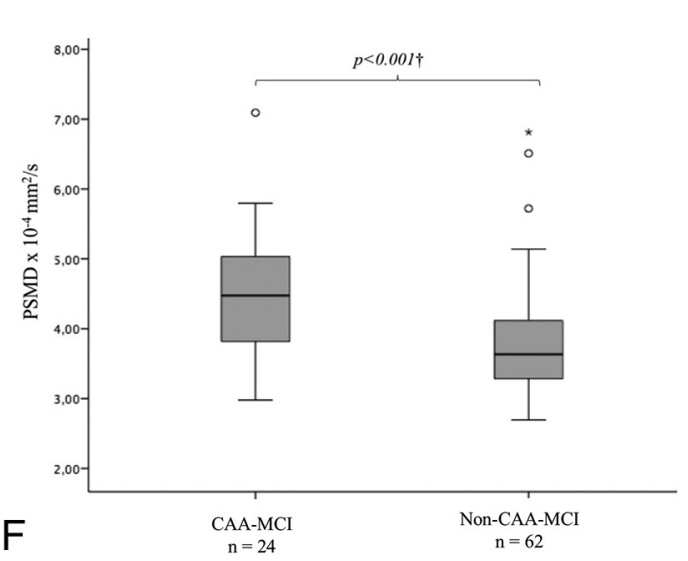

FIG 1. FLAIR images from a subject with CAA-MCI $(A)$ and one with a non-CAA-MCI (B), demonstrating different burdens of WMH. MD maps display the skeletonized WM tracts from the same subjects with CAA-MCI $(C)$ and non-CAA-MCI (D). E, Histograms depict the MD values of the voxels contained in the WM tract skeleton from the same subjects with CAA-MCI (solid line) and non-CAA-MCI (dashed line). $F$, The boxplot represents group differences in PSMD between CAA-MCI and non-CAA-MCI. The dagger indicates the results derived from ANCOVA, adjusting for age at MRI ( $P<.001)$.

anisotropy value of 0.2 ). MD images were projected onto the skeleton, using the fractional anisotropy-derived projection parameters. Next, to avoid contamination of the skeleton through CSF partial volume effects, we further masked the MD skeleton with a standard skeleton thresholded at a fractional anisotropy value of 0.3 and a mask provided with the PSMD pipeline to exclude regions adjacent to the ventricles, such as the fornix. Finally, PSMD was calculated as the difference between the 95th and fifth percentiles of the MD values of voxels contained within the skeleton. ${ }^{13}$ To ensure that results were not driven by outliers, we identified extreme PSMD (values below $1.5 \times$ IQR from the first quartile or above 1.5 $\times$ IQR from the third quartile) and excluded them from analyses.

\section{Neuroimaging Markers of cSVD}

MRI markers of cSVD were quantified by investigators blinded to all clinical data and according to the Standards for Reporting Vascular Changes on Neuroimaging (STRIVE) recommendations. ${ }^{27}$

The presence, number, and location of $\mathrm{CMB}$ were evaluated on the SWI according to the current consensus criteria. ${ }^{27,28}$ They were classified as lobar when located in cortical and corticosubcortical areas. cSS was visually assessed according to recently proposed criteria and transformed into a dichotomous variable (absence versus presence). ${ }^{29}$ Perivascular spaces in the centrum semiovale (CSOPVS) were rated on axial T1-weighted MRI, according to a previously developed score, ${ }^{30}$ and were analyzed as both dichotomous $(\leq 2$ versus $>2$ ) and ordinal variables. CMB, cSS, and CSO-PVS were analyzed by 2 experienced raters (N.R. and D.S.) using validated scales, and final ratings were obtained via a consensus.

After visual inspection of MR image quality, WMH volume, total brain volume, and total intracranial volume were calculated using FreeSurfer, Version 5.3 (http://surfer.nmr.mgh.harvard. edu), as previously described. ${ }^{31}$ Normalized total brain volume (nTBV) was calculated as the total brain volume/intracranial volume ratio, and normalized WMH volume (nWMHV) was calculated as WMH volume/intracranial volume $\times 100$.

\section{Statistical Analysis}

We compared the clinical and imaging characteristics between subjects with MCI with and without CAA using the $\chi^{2}$ or Fisher test for categoric variables and an independent $t$ test or MannWhitney $U$ test for continuous variables, as appropriate. The distribution of continuous variables was tested for normality with the Shapiro-Wilk test.

Log-transformed PSMD values were compared between patients with CAA-MCI and those with non-CAA-MCI using ANOVA, adjusted for age. We further adjusted for log-transformed nWMHV and cognitive status (MMSE).

Receiver operating characteristic curve analyses were used to quantify the performance of PSMD and nWMHV in discriminating subjects with CAA from those with non-CAA.

The association between PSMD and MRI markers of CSVD and PSMD and cognitive performances was evaluated in CAA- 


\begin{tabular}{|c|c|c|c|}
\hline & CAA-MCI $(n=24)$ & non-CAA-MCI $(n=62)$ & $P$ Value \\
\hline \multicolumn{4}{|l|}{ Demographics } \\
\hline Age at NPT (mean) [SD] (yr) & $74.73[5.99]$ & $73.25[8.33]$ & .36 \\
\hline Female (No.) (\%) & $7(29.2)$ & $26(41.9)$ & .27 \\
\hline Education (median) (IQR) (yr) & $16(16,18)$ & $16(14,18)$ & $.04^{\mathrm{a}}$ \\
\hline \multicolumn{4}{|l|}{ Vascular risk factors } \\
\hline Hypertension (No.) (\%) & $13(54.2)$ & $40(64.5)$ & .38 \\
\hline Diabetes (No.) (\%) & $1(4.2)$ & $10(16.1)$ & .17 \\
\hline Atrial fibrillation (No.) (\%) & $4(16.7)$ & $5(8.1)$ & .26 \\
\hline Dyslipidemia (No.) (\%) & $13(54.2)$ & $44(71.0)$ & .14 \\
\hline \multicolumn{4}{|l|}{ Neuropsychological performances } \\
\hline MMSE (median) (IQR) & $25.5(24-28)$ & $28(26-29)$ & $.006^{\mathrm{a}}$ \\
\hline MMSE (z score) (median) (IQR) & $-1.88(-3.28,-0.16)$ & $0(-1.48-1.11)$ & $.003^{\mathrm{a}}$ \\
\hline Memory (z score) (median) (IQR) & $-1.91(-2.38,-0.75)$ & $-0.47(-1.60-0.56)$ & $.005^{\mathrm{a}}$ \\
\hline Processing speed (z score) (median) (IQR) & $-0.24(-0.62-0.15)$ & $-0.11(-0.46-0.27)$ & .34 \\
\hline Language (z score) (median) (IQR) & $-0.44(-0.91-0.36)$ & $-0.36(-1.17-0.19)$ & .95 \\
\hline Executive function ( $z$ score) (median) (IQR) & $-0.59(-1.89-0.22)$ & $-0.21(-0.86-0.12)$ & .20 \\
\hline \multicolumn{4}{|l|}{ Imaging } \\
\hline $\operatorname{PSMD}\left(\times 10^{-4} \mathrm{~mm}^{2} / \mathrm{s}\right)$ (median) (IQR) & $4.48(3.81-5.09)$ & $3.63(3.28-4.13)$ & $<.001^{\mathrm{a}}$ \\
\hline Lobar CMB count (median) (IQR) & $5.5(3.0-24.50)$ & $0(0-0)$ & $<.001^{\mathrm{a}}$ \\
\hline cSS (presence) (No.) (\%) & $9(37.5)$ & $0(0.0)$ & $<.001^{\mathrm{a}}$ \\
\hline High CSO-PVS score $(>2)(\mathrm{No}).(\%)$ & $11(45.8)$ & $3(4.8)$ & $<.001^{\mathrm{a}}$ \\
\hline nWMHV (median) (IQR) & $0.42(0.28-1.74)$ & $0.29(0.14-0.75)$ & $.02^{\mathrm{a}}$ \\
\hline nTBV (mean) [SD] & $0.61[0.04]$ & $0.64[0.05]$ & $.004^{\mathrm{a}}$ \\
\hline
\end{tabular}

Note:-NPT indicates neuropsychological tests.

a Significant.

MCI and non-CAA-MCI separately. Linear regression models were used to explore relationships between PSMD and structural MRI markers of cSVD (lobar CMB count, CSO-PVS score, the presence of cSS, nWMHV, and nTBV), adjusting for age. The association between PSMD and cognitive scores in each domain was explored using linear regression models in both groups separately, adjusting for structural MRI markers of cSVD and the time delay between the neuropsychological evaluation and the MRI. Because cognitive scores were already adjusted for age, sex, and education level, these variables were not included in the models.

The statistical significance level was set at .05 for all analyses. We used the Statistical Package for the Social Sciences, Version 24.0 for Windows (IBM) and R (v3.5.3) for statistical analysis.

\section{RESULTS}

We identified 134 subjects with cognitive impairment enrolled in this prospective study who underwent a research MRI. Of them, 42 subjects were excluded on the basis of the prespecified criteria: diagnosis of dementia $(n=6)$, possible CAA category $(n=10)$, presence of deep CMB $(n=5)$, lack of neuropsychological tests $(n=19)$, and the presence of excessive motion artifacts on DTI, based on qualitative visual inspection $(n=2)$. Additionally, 3 outliers with extreme PSMD values (all with high values) were identified in each group and were excluded from the analysis. The final cohort consisted of 86 subjects with MCI (mean age, 73.7 [SD , 7.7] years; $38.4 \%$ female) without a history of ischemic stroke or ICH, including 24 subjects with probable CAA (CAAMCI; 27.9\%) and 62 without CAA (non-CAA-MCI; 72.1\%).

\section{Comparison between Subjects with CAA and Those with Non-CAA}

Subjects with CAA-MCI and non-CAA-MCI were similar in age and vascular risk factors (Table 1). MMSE scores were lower in subjects with CAA-MCI compared with subjects with non-CAAMCI $(P=.003)$. Patients with CAA-MCI had worse performance in memory than subjects with non-CAA-MCI $(P=.005)$. The 2 groups had similar scores across all other cognitive domains $(P>$ .05 , for all). Compared with those with non-CAA-MCI, subjects with CAA-MCI presented with a higher burden of MRI markers of cSVD, including a higher prevalence of cSS $(P<.001)$, higher lobar CMB count $(P<.001)$, greater nWMHV $(P=.016)$, a higher prevalence of high CSO-PVS scores $(P<.001)$, and lower nTBV $(P=.004)$. PSMD values were significantly higher in CAA-MCI in comparison with non-CAA-MCI $(P<.001)$ (Fig $1 F)$, even after adjusting for age $(P<.001)$. In a post hoc analysis, we found that PSMD remained significantly higher in subjects with CAA-MCI compared with subjects with non-CAA-MCI when further controlling for nWMHV $(P=.007)$ or cognitive status (MMSE $z$ scores $)(P<.001)$. In receiver operating characteristic analyses, PSMD (area under the curve $=0.755$; 95\% CI, $0.636-0.873 ; P<.001$ ) was able to significantly discriminate subjects with CAA from those without CAA, and it yielded a greater area under the curve than nWMHV (area under the curve = 0.668; 95\% CI, 0.544-0.792; $P=.016$ ) (Online Supplemental Data).

\section{Associations between PSMD and Markers of cSVD}

In linear regression analyses adjusted for age, increased PSMD was associated with greater nWMHV both in CAA-MCI $(\beta=0.75$; $P<.001)$ and non-CAA-MCI $(\beta=0.69 ; P<.001)$ groups, but not with nTBV, CMB, CSO-PVS, or cSS (Table 2). In multiple regression models including all quantified structural MRI markers of CSVD, only nWMHV remained independently associated with PSMD in subjects with CAA-MCI $(\beta=0.66 ; P<.001)$ and non-CAA-MCI $(\beta=0.71 ; P<.001)$ (Table 2 and Online Supplemental Data). 
Table 2: Association between PSMD and MRI markers of small-vessel disease in subjects with CAA-MCI and non-CAA-MCl ${ }^{\mathrm{a}}$

\begin{tabular}{|c|c|c|c|c|c|c|c|c|}
\hline \multirow{3}{*}{ CAA-MCI $(n=24)$} & \multicolumn{4}{|c|}{ Model $1^{\mathrm{b}}$} & \multicolumn{4}{|c|}{ Model $2^{c}$} \\
\hline & \multirow[t]{2}{*}{ Std. $\beta$} & \multicolumn{2}{|c|}{$95 \% \mathrm{Cl}$} & \multirow[t]{2}{*}{$P$} & \multirow[t]{2}{*}{ Std. $\beta$} & \multicolumn{2}{|c|}{$95 \% \mathrm{Cl}$} & \multirow[t]{2}{*}{$P$} \\
\hline & & & & & & & & \\
\hline Lobar CMB count & 0.15 & -0.27 & 0.58 & .46 & 0.15 & -0.13 & 0.42 & .28 \\
\hline cSS & 0.27 & -0.15 & 0.70 & .20 & 0.12 & -0.16 & 0.40 & .38 \\
\hline CSO-PVS score & 0.31 & -0.09 & 0.71 & .13 & 0.20 & -0.10 & 0.49 & .18 \\
\hline nWMHV & 0.75 & 0.49 & 1.02 & $<.001^{d}$ & 0.66 & 0.37 & 0.95 & $<.001^{\mathrm{d}}$ \\
\hline nTBV & -0.26 & -0.79 & 0.26 & .31 & -0.17 & -0.54 & 0.20 & .34 \\
\hline \multicolumn{9}{|c|}{ Non-CAA-MCI $(n=62)$} \\
\hline CSO-PVS & -0.02 & -0.24 & 0.21 & .87 & 0.10 & -0.07 & 0.27 & .25 \\
\hline nWMHV & 0.69 & 0.50 & 0.89 & $<.001^{\mathrm{d}}$ & 0.71 & 0.51 & 0.91 & $<.001^{\mathrm{d}}$ \\
\hline nTBV & -0.23 & -0.51 & 0.06 & .11 & -0.14 & -0.36 & 0.08 & .20 \\
\hline
\end{tabular}

Note:-Std. $\beta$ indicates standardized beta coefficient.

${ }^{a}$ Linear regression models with PSMD $\left(\times 10^{-4} \mathrm{~mm}^{2} / \mathrm{s}\right)$ as the dependent variable.

${ }^{\mathrm{b}}$ Simple Linear regression analyses adjusted for age.

${ }^{c}$ Multiple regression models, including all neuroimaging markers and adjusted for age. In subjects with non-CAA-MCI, the presence of cSS and lobar CMB count was automatically excluded from the models due to the absence of variance within the group.

d Significant.

Table 3: Association between PSMD and cognitive performance in subjects with CAA-MCl and non-CAA-MCl ${ }^{\mathrm{a}}$

\begin{tabular}{|c|c|c|c|c|}
\hline & \multicolumn{4}{|c|}{ Adjusted Model ${ }^{\mathrm{b}}$} \\
\hline & Std. $\boldsymbol{\beta}$ & \multicolumn{2}{|c|}{$95 \% \mathrm{Cl}$} & $P$ Value \\
\hline \multicolumn{5}{|l|}{ CAA-MCI $(n=24)$} \\
\hline Global cognitive status & -0.40 & -1.21 & 0.41 & .31 \\
\hline Memory & -0.69 & -1.52 & 0.15 & .10 \\
\hline $\begin{array}{l}\text { Processing speed/ } \\
\text { attention }\end{array}$ & -1.08 & -1.76 & -0.40 & $.004^{c}$ \\
\hline Language & -0.47 & -1.16 & 0.21 & .16 \\
\hline Executive function & -0.64 & -1.37 & 0.10 & .09 \\
\hline \multicolumn{5}{|l|}{ non-CAA-MCI $(n=62)$} \\
\hline Global cognitive status & 0.30 & -0.13 & 0.72 & .17 \\
\hline Memory & 0.14 & -0.28 & 0.56 & .50 \\
\hline $\begin{array}{l}\text { Processing speed/ } \\
\text { attention }\end{array}$ & 0.16 & -0.27 & 0.59 & .46 \\
\hline Language & 0.14 & -0.29 & 0.57 & .52 \\
\hline Executive function & 0.11 & -0.30 & 0.53 & .58 \\
\hline \multicolumn{5}{|c|}{$\begin{array}{l}\text { a Multiple regression models with cognitive performance as the dependent variable. } \\
{ }^{b} \text { Models include PSMD, nWMHV, nTBV, CSO-PVS score, the presence of cSS and } \\
\text { lobar CMB count; adjusting for the time delay between the neuropsychological } \\
\text { evaluation and the MRI. In subjects with non-CAA-MCI, the presence of cSS and } \\
\text { the lobar CMB count were automatically excluded from the models due to an ab- } \\
\text { sence of variance within the group. } \\
{ }^{c} \text { Significant. }\end{array}$} \\
\hline
\end{tabular}

\section{Associations between PSMD and Cognitive Function}

In the CAA-MCI group, multiple regression models accounting for lobar CMB count, cSS, CSO-PVS score, nWMHV, and nTBV demonstrated that increased PSMD was independently associated with worse performance in processing speed $(\beta=-1.08 ; P=$ .004) (Table 3 and Online Supplemental Data). In the non-CAAMCI group, multiple regression analyses did not reveal any significant associations between PSMD and scores reflecting each cognitive domain (Table 3 and Online Supplemental Data).

\section{DISCUSSION}

Several key findings emerge from this study on PSMD in patients with CAA presenting with MCI in the absence of ICH. First, subjects with MCI with CAA showed increased PSMD values compared with subjects with MCI without CAA, even after adjusting for baseline differences in age, nWMHV, and cognitive status.
Second, we confirmed that PSMD was strongly associated with WMHV in our CAA population, but not with other structural markers of cSVD. Third, we found that PSMD values were associated with worse performance in processing speed among subjects with CAA-MCI after controlling for the presence of other MRI markers of cSVD. In contrast, PSMD was not associated with cognitive function in subjects with non-CAA-MCI.

PSMD studies have, so far, focused mainly on community-dwelling $^{13,32,33}$ and cognitively impaired elderly subjects, ${ }^{13,34}$ as well as those with inherited ${ }^{13,35}$ and sporadic cSVD. ${ }^{13,36}$ To our knowledge, only 1 previous study has investigated the performance of PSMD in sporadic CAA, including subjects with and without $\mathrm{ICH}$ recruited from both stroke-prevention and memory clinics. ${ }^{37}$ Because CAA pathology is highly prevalent and significantly contributes to vascular cognitive impairment in the elderly population, ${ }^{38}$ further investigating the performance of PSMD in the context of CAA is an important step for the validation of this new neuroimaging biomarker as a surrogate for cognitive dysfunction in CSVD. ${ }^{39}$

As expected, the PSMD values we obtained in subjects with CAA in a memory clinic were remarkably similar to those found in other sporadic cSVD cohorts, ${ }^{13,36}$ including another CAA cohort, ${ }^{37}$ corroborating the reproducibility and stability of PSMD across different scanners, sequences, and even clinical samples. ${ }^{13,40}$

The observed increase in PSMD values among subjects with CAA-MCI supports the hypothesis that whole-brain microstructural integrity is impaired in this population. Our results are in accordance with previous studies showing microstructural abnormalities in CAA when relying on other DTI-based methods. ${ }^{11,41}$ Most important, PSMD offers several advantages in comparison with other DTI methods: It is a fully automated and fast technique; it offers higher interscanner reproducibility; power calculations have shown smaller sample size estimates for PSMD; and it is more strongly associated with performance in processing speed. ${ }^{13}$

Group differences in PSMD remained significant (CAA-MCI versus non-CAA-MCI) even after adjusting for age, nWMHV, and MMSE scores. This finding suggests that PSMD differences are not solely driven by these factors and may indicate that this marker, like other global DTI measures, might capture abnormalities not visible on structural MRI sequences. 
In our CAA-MCI sample, PSMD was strongly associated with nWMHV, but not with hemorrhagic markers of CAA (lobar CMBs and cSS), a finding in line with those from a recent study on a different CAA sample, ${ }^{37}$ and this finding suggests that white matter tract disruption in CAA may be more closely linked to cSVD damage from ischemic rather than hemorrhagic origin.

The encouraging finding that PSMD is independently associated with processing speed in our subjects with CAA-MCI, after adjusting for other conventional MRI markers of cSVD, is in consonance with recently published results from another CAA sample. ${ }^{37}$ The lack of association between PSMD and cognition in the non-CAA-MCI sample is consistent with findings from other studies in cohorts with low burdens of cSVD. ${ }^{13,36}$ PSMD, like other DTI metrics, appears to be more sensitive to cSVD-related white matter abnormalities than to neurodegenerative pathology. ${ }^{13,42}$ The low burden of cSVD pathology observed in our non-CAA sample might explain the absence of association between PSMD and processing speed.

Our results argue in favor of a strong link between PSMD and processing speed in cSVD populations, as advocated in the original PSMD study. ${ }^{13}$ However, mechanisms underlying these strong associations with cognition are incompletely understood. McCreary et $\mathrm{al}^{37}$ reported that a greater variation in white matter MD could be seen in microarchitectural disruption caused by pathologic processes. Although the histopathologic features specifically associated with increases in PSMD remain unknown, tissue rarefaction and lower myelin density have been related to MD variations in subjects with CAA. ${ }^{43}$ It is possible that similar microstructural abnormalities underlie changes in PSMD in CAA, reflecting disruption of synaptic transmission, which could affect cognition.

Our study has limitations. The small sample size of our cohort may account for the relatively weak cognitive correlations observed. Hence, our findings should be considered preliminary and require external validation in larger CAA cohorts. By including only subjects with MCI (cognitively healthy and subjects with CAA and dementia were excluded), our study was not designed to assess relationships between PSMD and the full spectrum of cognitive impairment, ranging from MCI to dementia. Still, our significant findings in subjects with mild forms of cognitive impairment argue in favor of the robustness of PSMD as a biomarker for cognitive function in CAA. Additionally, our study included participants with a specific presentation of CAA (ie, mild cognitive symptoms without ICH). We excluded subjects with ICH because this likely represents a different phenotype of the disease. ${ }^{44}$ While we designed our study to examine this specific group of subjects with CAA who frequently present in memory clinic settings, our results cannot be generalized to other CAA populations or phenotypes. Another limitation of our study is the absence of comparisons between PSMD and other previously validated DTI-based markers to assess whether this new method constitutes a superior biomarker.

Nonetheless, this study also has several strengths and expands on previous literature by evaluating the relevance of PSMD in a specific phenotype of CAA and investigating its independent cognitive and neuroimaging associations.

\section{CONCLUSIONS}

PSMD values are higher among cognitively impaired subjects with CAA in comparison with those without CAA and are associated with nWMHV and performance in processing speed. Our preliminary results support the relevance of PSMD, a completely automated DTI-based method, in capturing microstructural brain changes in subjects with CAA, even in the absence of ICH. PSMD may serve as a biomarker in future clinical trials involving CAA and other cSVD.

Disclosures: Nicolas Raposo- RELATED: Grant. Fulbright Scholarship, Arthur Sachs Scholarship from the Harvard University Committee on General Scholarship, and a Philippe Foundation research grant; UNRELATED: Travel/Accommodations/Meeting Expenses Unrelated to Activities Listed: Pfizer, Bristol Myers Squibb, Bayer. Maria Clara Zanon Zotin-RELATED: Support for Travel to Meetings for the Study or Other Purposes: Mass General Brigham, Comments: support to travel to a conference. Dorothee Schoemaker-RELATED: Grant: American Heart Association, Comments: postdoctoral fellowship.* Kristin Schwab_UNRELATED: Employment: Massachusetts General Hospital MarkVCID. Markus D. Schirmer-UNRELATED: Grants/Grants Pending: European Research Council.* Mark Etherton-UNRELATED: Grants/Grants Pending: American Academy of Neurology.* Steven GreenbergRELATED: Grant: National Institutes of Health; UNRELATED: Consultancy: Bayer, Biogen, IQVIA, Roche, Comments: Data Safety Monitoring Boards; Grants/Grants Pending: National Institutes of Health; Royalties: UpToDate. Marco DueringRELATED: Grant: Else Kröner-Fresenius-Stiftung*; UNRELATED: Payment for Lectures Including Service on Speakers Bureaus: Bayer Vital, Pfizer, Comments: Lecture honoraria paid to individual author. Anand Viswanathan-RELATED: Grant: National Institutes of Health*; UNRELATED: Consultancy: Biogen, Alynylam Pharmaceuticals, Roche. *Money paid to the institution.

\section{REFERENCES}

1. Charidimou A, Gang Q, Werring DJ. Sporadic cerebral amyloid angiopathy revisited: recent insights into pathophysiology and clinical spectrum. J Neurol Neurosurg Psychiatry 2012;83:124-37 CrossRef Medline

2. Planton M, Saint-Aubert L, Raposo N, et al. High prevalence of cognitive impairment after intracerebral hemorrhage. PLoS One 2017;12:e01778886 CrossRef Medline

3. Boyle PA, Yu L, Nag S, et al. Cerebral amyloid angiopathy and cognitive outcomes in community-based older persons. Neurology 2015;85:1930-36 CrossRef Medline

4. Heinen R, Vlegels N, de Bresser J, et al. The cumulative effect of small vessel disease lesions is reflected in structural brain networks of memory clinic patients. Neuroimage Clin 2018;19:963-69 CrossRef Medline

5. O'Sullivan M, Jones DK, Summers PE, et al. Evidence for cortical "disconnection" as a mechanism of age-related cognitive decline. Neurology 2001;57:632-38 CrossRef Medline

6. Martinez-Ramirez S, Greenberg SM, Viswanathan A. Cerebral microbleeds: overview and implications in cognitive impairment. Alzheimers Res Ther 2014;6:1-7 CrossRef Medline

7. Charidimou A, Ni J, Ramirez Martinez S, et al. Cortical superficial siderosis in memory clinic patients: further evidence for underlying cerebral amyloid angiopathy. Cerebrovasc Dis 2016;41:156-62 CrossRef Medline

8. Smith EE, Gurol ME, Eng JA, et al. White matter lesions, cognition, and recurrent hemorrhage in lobar intracerebral hemorrhage. Neurology 2004;63:1606-12 CrossRef Medline

9. Xiong L, Van Veluw SJ, Bounemia N, et al. Cerebral cortical microinfarcts on magnetic resonance imaging and their association with cognition in cerebral amyloid angiopathy. Stroke 2018;49:2330-36 CrossRef Medline

10. Charidimou A, Boulouis G, Gurol ME, et al. Emerging concepts in sporadic cerebral amyloid angiopathy. Brain 2017;140:1829-50 CrossRef Medline

11. Reijmer YD, Fotiadis P, Martinez-Ramirez S, et al. Structural network alterations and neurological dysfunction in cerebral amyloid angiopathy. Brain 2015;138:179-88 CrossRef Medline 
12. Charidimou A, Boulouis G, Haley K, et al. White matter hyperintensity patterns in cerebral amyloid angiopathy and hypertensive arteriopathy. Neurology 2016;86:505-11 CrossRef Medline

13. Baykara E, Adam R, Tuladhar AM, et al. A novel imaging marker for small vessel disease based on skeletonization of white matter tracts and diffusion histograms. Ann Neurol 2016;40:581-92 CrossRef Medline

14. Petersen RC. Mild cognitive impairment as a diagnostic entity. $J$ Intern Med 2004;256:183-94 CrossRef Medline

15. Linn J, Halpin A, Demaerel P, et al. Prevalence of superficial siderosis in patients with cerebral amyloid angiopathy. Neurology 2010;74:1346-50 CrossRef Medline

16. Crum RM, Anthony JC, Bassett SS, et al. Population-based norms for the Mini-Mental State Examination by age and educational level. JAMA 1993;269:2386-91 CrossRef Medline

17. Weintraub S, Salmon D, Mercaldo N, et al. The Alzheimer's Disease Centers' Uniform Data Set (UDS): the neuropsychologic test battery. Alzheimer Dis Assoc Disord 2009;23:91-101 CrossRef Medline

18. Corrigan JD, Hinkeldey NS. Relationships between parts A and B of the Trail Making Test. J Clin Psychol 1987;43:402-09 CrossRef Medline

19. Joy S, Kaplan E, Fein D. Speed and memory in the WAIS-III Digit Symbol-Coding subtest across the adult lifespan. Arch Clin Neuropsychol 2004;19:759-67 CrossRef Medline

20. Sánchez-Cubillo I, Periáñez JA, Adrover-Roig D, et al. Construct validity of the Trail Making Test: role of task-switching, working memory, inhibition/interference control, and visuomotor abilities. J Int Neuropsychol Soc. 2009;15:438-50 CrossRef Medline

21. Brandt J. The Hopkins Verbal Learning Test: development of a new memory test with six equivalent forms. Clin Neuropsychol 1991;5:125-42 CrossRef

22. Wechsler D. Wechsler Memory Scale-Revised Manual. Harcourt Brace Jovanovich; 1987

23. Mack WJ, Freed DM, Williams BW, et al. Boston Naming Test: shortened versions for use in Alzheimer's disease. J Gerontol 1992;47:P154-58 CrossRef Medline

24. Tombaugh T, Kozak J, Rees L. Normative data stratified by age and education for two measures of verbal fluency FAS and animal naming. Arch Clin Neuropsychol 1999;14:167-77 Medline

25. Fastenau PS, Denburg NL, Mauer BA. Parallel short forms for the Boston Naming Test: psychometric properties and norms for older adults. J Clin Exp Neuropsychol 1998;20:828-34 CrossRef Medline

26. Tombaugh T. Trail Making Test A and B: normative data stratified by age and education. Arch Clin Neuropsychol. 2004;19:203-14 CrossRef Medline

27. Wardlaw JM, Smith EE, Biessels GJ, et al. Neuroimaging standards for research into small vessel disease and its contribution to ageing and neurodegeneration. Lancet Neurol 2013;12:822-38 CrossRef Medline

28. Greenberg SM, Vernooij MW, Cordonnier C, et al. Cerebral microbleeds: a field guide to their detection and interpretation. Lancet Neurol 2009;8:165-74 CrossRef Medline

29. Charidimou A, Linn J, Vernooij MW, et al. Cortical superficial siderosis: detection and clinical significance in cerebral amyloid angiopathy and related conditions. Brain 2015;138:2126-39 CrossRef Medline

30. Potter GM, Chappell FM, Morris Z, et al. Cerebral perivascular spaces visible on magnetic resonance imaging: development of a qualitative rating scale and its observer reliability. Cerebrovasc Dis 2015;39:224-31 CrossRef Medline

31. Fotiadis P, van Rooden S, van der Grond J, et al. Cortical atrophy in patients with cerebral amyloid angiopathy: a case-control study. Lancet Neurol 2016;15:811-19 CrossRef Medline

32. Deary IJ, Ritchie SJ, Muñoz Maniega S, et al. Brain peak width of skeletonized mean diffusivity (PSMD) and cognitive function in later life. Front Psychiatry 2019;10:524 CrossRef Medline

33. Lam BY, Leung KT, Yiu B, et al. Peak width of skeletonized mean diffusivity and its association with age-related cognitive alterations and vascular risk factors. Alzheimers Dement (Amst) 2019;11:721-29 CrossRef Medline

34. Low A, Mak E, Stefaniak JD, et al. Peak width of skeletonized mean diffusivity as a marker of diffuse cerebrovascular damage. Front Neurosci 2020;14:238 CrossRef Medline

35. Schouten TM, de Vos F, van Rooden S, et al. Multiple approaches to diffusion magnetic resonance imaging in hereditary cerebral amyloid angiopathy mutation carriers. J Am Heart Assoc 2019;8: e011288 CrossRef Medline

36. Wei N, Deng Y, Yao L, et al. A neuroimaging marker based on diffusion tensor imaging and cognitive impairment due to cerebral white matter lesions. Front Neurol 2019;10:1-7 CrossRef Medline

37. McCreary CR, Beaudin AE, Subotic A, et al. Cross-sectional and longitudinal differences in peak skeletonized white matter mean diffusivity in cerebral amyloid angiopathy. Neuroimage Clin 2020;27:102280 CrossRef Medline

38. Viswanathan A, Greenberg SM. Cerebral amyloid angiopathy in the elderly. Ann Neurol 2011;70:871-80 CrossRef

39. Smith EE, Biessels GJ, De Guio F, et al. Harmonizing brain magnetic resonance imaging methods for vascular contributions to neurodegeneration. Alzheimers Dement (Amst) 2019;11:191-204 CrossRef Medline

40. Beaudet G, Tsuchida A, Petit L, et al. Age-related changes of peak width skeletonized mean diffusivity (PSMD) across the adult lifespan: a multi-cohort study. Front Psychiatry 2020;11:342 CrossRef Medline

41. Salat DH, Smith EE, Tuch DS, et al. White matter alterations in cerebral amyloid angiopathy measured by diffusion tensor imaging. Stroke 2006;37:1759-64 CrossRef Medline

42. Finsterwalder S, Vlegels N, Gesierich B, et al. Small vessel disease more than Alzheimer's disease determines diffusion MRI alterations in memory clinic patients. Alzheimers Dement 2020:16:504-14 CrossRef Medline

43. van Veluw SJ, Reijmer YD, van der Kouwe AJ, et al. Histopathology of diffusion imaging abnormalities in cerebral amyloid angiopathy. Neurology 2019;92:43 CrossRef Medline

44. Charidimou A, Martinez-Ramirez S, Shoamanesh A, et al. Cerebral amyloid angiopathy with and without hemorrhage: evidence for different disease phenotypes. Neurology 2015;84:1206-12 CrossRef 